\title{
Fatores associados à mortalidade por causas inespecíficas e mal definidas no estado do Amazonas, Brasil, de 2006 a 2012
}

\author{
Factors associated with unspecified and ill-defined causes of death \\ in the State of Amazonas, Brazil, from 2006 to 2012
}

Patrícia Carvalho da Silva Balieiro (https://orcid.org/0000-0002-5221-6319) ${ }^{1}$ Leila Cristina Ferreira da Silva (https://orcid.org/0000-0003-0649-4139) ${ }^{1}$ Vanderson de Souza Sampaio (https://orcid.org/0000-0001-7307-8851) ${ }^{1}$ Eyrivania Xavier do Monte (https://orcid.org/0000-0002-3712-0239) ${ }^{1}$ Edylene Maria dos Santos Pereira (https://orcid.org/0000-0002-6049-5469) ${ }^{1}$ Lais Araújo Ferreira de Queiroz (https://orcid.org/0000-0003-3785-0028) ${ }^{1}$ Rita Saraiva (https://orcid.org/0000-0003-4533-084X) ${ }^{1}$ Antonio José Leal Costa (https://orcid.org/0000-0002-0402-4865) ${ }^{2}$
${ }^{1}$ Núcleo de Sistemas de Informações, Fundação de Vigilância em Saúde do Amazonas. Av. Torquato Tapajós, Colônia Santo Antônio. 69093-018 Manaus AM Brasil. tricia.cks@gmail.com ${ }^{2}$ Instituto de Estudos em Saúde Coletiva, Universidade Federal do Rio de Janeiro. Rio de Janeiro RJ Brasil.

\begin{abstract}
This study aimed to investigate factors associated with unspecified and ill-defined causes of death in the State of Amazonas (AM), Brazil. This is a cross-sectional study on 90,439 non-fetal deaths of residents in AM from 2006 to 2012. The hierarchical multinomial logistic model estimated odds ratios of unspecified and ill-defined causes of death. Ill-defined and unspecified causes of death proportional mortality was, respectively, 16.6\% and $9.1 \%$. Ill-defined causes showed a decreasing trend over the years, while unspecified causes only decreased in the last two years. Unspecified causes of death were associated with residence and death outside the capital, public roads, female gender, age group 10-49 years, brown skin color and when certified by forensic doctors. Ill-defined causes of death were associated with residence and occurrence outside capital, at home, ages 40 years and older, non-whites, not being single, low schooling, under medical care and when examiner was unknown. Ill-defined and unspecified cause mortality in the State of Amazonas decreased between 2006 and 2012 in AM and was associated with space and time, demographic and socioeconomic factors and medical care at the moment of death. Key words Mortality, Death certificate, Underlying cause of death, Health information systems, International classification of diseases
\end{abstract}

Resumo Objetivou-se investigar fatores associados à mortalidade por causas inespecíficas e mal definidas no estado do Amazonas (AM). Desenvolveu-se um estudo seccional incluindo 90.439 registros de óbitos não fetais, com residência e ocorrência no AM entre 2006 e 2012. Foram estimadas razões de chances de causas inespecíficas e mal definidas por meio de regressão logística multinomial hierárquica. A proporção de causas mal definidas e inespecíficas foi, respectivamente, $16,6 \%$ e 9,1\%. A ocorrência de causas mal definidas diminuiu ao longo dos anos e a de causas inespecíficas somente no último biênio. As causas inespecíficas associaram-se com residência e ocorrência do óbito fora da capital, via pública, sexo feminino, dos 10 aos 49 anos, cor parda e quando atestadas por legistas. As causas mal definidas associaram-se com residência e ocorrência fora da capital, em domicílios, a partir de 40 anos, cor não branca, não ser solteiro, baixa escolaridade, assistência médica e falta de informação sobre o atestante. A mortalidade por causas mal definidas e inespecíficas no AM declinou entre 2006 e 2012, associando-se às dimensões espacial e temporal, fatores demográficos, socioeconômicos e à assistência médica na ocasião do óbito.

Palavras-chave Mortalidade, Atestado de óbito, Causa básica de morte, Sistemas de informação em saúde, Classificação internacional de doenças. 


\section{Introdução}

Os registros de mortalidade são tradicionalmente utilizados na elaboração de indicadores de saúde. No Brasil, tais registros estão disponíveis no Sistema de Informações sobre Mortalidade (SIM) do Ministério da Saúde, criado em 1975. Os dados do SIM têm sua origem na Declaração de Óbito (DO) - documento padronizado em todo o território nacional - preenchida pelos médicos para cada caso de morte ocorrida no país ${ }^{1}$.

Tendo em vista a importância dos indicadores de saúde baseados em medidas de mortalidade no contexto da saúde pública, a avaliação dos sistemas de informações sobre mortalidade tem sido objeto de estudos em todo o mundo ${ }^{2-4}$, em especial no que diz respeito à abrangência dos sistemas e à qualidade dos dados. A informação sobre a causa básica do óbito é particularmente relevante. A descrição do perfil de mortalidade de uma população segundo a causa básica orienta a aplicação e a avaliação de medidas preventivas, portanto, a sua correta classificação é imprescindível ${ }^{5,6}$.

A proporção de óbitos por causas mal definidas, agrupadas no capítulo XVIII da décima revisão da Classificação Estatística Internacional de Doenças e Problemas Relacionados à Saúde (CID10) sob o título "Sintomas, sinais e achados anormais de exames clínicos e de laboratório não classificados em outra parte", tem sido tradicionalmente utilizada como indicador para avaliação da qualidade das informações de mortalidade por causas ${ }^{7}$. A exatidão dos dados de mortalidade por causas também pode ser avaliada por meio da enumeração dos diagnósticos incompletos ou causas inespecíficas. São considerados diagnósticos incompletos as consequências ou complicações da causa básica de morte - como, por exemplo, a septicemia, as insuficiências cardíaca, renal, hepática etc. - e as doenças de órgãos declaradas como cardiopatia, hepatopatia e nefropatia. Diferentemente das causas mal definidas, as inespecíficas estão distribuídas por toda a CID-10, correspondendo aos chamados códigos residuais de diversos capítulos ${ }^{8}$.

Quando considerados em conjunto, a proporção de óbitos por causas mal definidas ou inespecíficas possibilita uma análise mais abrangente da qualidade da informação sobre mortalidade, particularmente no que diz respeito à exatidão dos dados sobre causas de morte ${ }^{9}$. A identificação dos fatores relacionados à ocorrência de óbitos por causas mal definidas e inespecíficas auxilia o direcionamento de esforços voltados para o aprimoramento dos registros de mortalidade.
Não obstante o SIM ser o sistema de informação em saúde mais avaliado no Brasil, os estudos realizados concentram-se em poucas regiões e estados do país ${ }^{10}$, não havendo referência a estudos voltados especificamente para a avaliação das informações sobre mortalidade no AM. O presente estudo teve como objetivo analisar os fatores associados à mortalidade por causas inespecíficas e mal definidas no estado do Amazonas, de 2006 a 2012.

\section{Métodos}

O estado do Amazonas está localizado na região Norte do Brasil, constituindo-se como a maior unidade federativa do país com uma área de 1.559.161 km2 dividida em 62 municípios. De acordo com o censo demográfico de 2010, possuía 3.483.985 habitantes com $79 \%$ da população vivendo em áreas urbanas e $21 \%$ nas zonas rurais, dos quais 1.802 .525 (52\%) residindo na capital Manaus ${ }^{11}$.

Desenvolveu-se um estudo seccional, exploratório, baseado em série de registros de óbitos do SIM, sob gerência do Núcleo de Sistemas de Informações da Fundação de Vigilância em Saúde do Amazonas - NUSI/FVS AM. A base de dados foi gerada em novembro de 2014. Foram incluídos os óbitos não fetais de residentes no Amazonas, ocorridos no próprio estado entre 2006 e 2012 e com informação sobre a causa básica.

A causa básica de morte originalmente registrada nas DO (variável causada do SIM) foi classificada como bem definida, inespecífica ou mal definida, com base na tipologia proposta por $\mathrm{Na}$ ghavi et al. ${ }^{12}$. As causas inespecíficas correspondem aos códigos "garbage" definidos no estudo "Global Burden of Disease", incluindo diagnósticos indefinidos ou incompletos, de uso limitado em saúde pública para fins de planejamento e avaliação de medidas de prevenção ${ }^{8,13}$. São subdivididas em quatro grupos compostos por códigos residuais da CID10, a saber: 1) causas que não podem ser causa básica (excluídas as mal definidas - capítulo XVIII);2) causas intermediárias; 3 ) causas terminais; e 4) causas não especificadas ${ }^{12}$. As causas mal definidas correspondem às categorias e subcategorias do capítulo XVIII da CID107.

Os municípios foram classificados em três categorias: capital, referência para as regionais de saúde e demais municípios ${ }^{14}$. Para fins de análise, a regional de saúde "Entorno de Manaus e Rio Negro" foi subdividida nas suas microrregionais e os municípios Manaus e São Gabriel da Ca- 
choeira foram classificados, respectivamente, nas categorias "capital" e "municípios de referência" (Figura 1).

Calculou-se a mortalidade proporcional por causas inespecíficas e mal definidas, total e segundo categorias das variáveis explicativas selecionadas $^{15}$. Foram identificadas as categorias (de três caracteres) da CID10 mais frequentes entre as causas inespecíficas e mal definidas, segundo tipo de município de ocorrência do óbito e para todo o estado. Em seguida foram estimadas razões de chances (odds ratios - OR) de mortalidade por causas inespecíficas e mal definidas, brutas e ajustadas por meio de modelo de regressão logística multinomial hierárquico ${ }^{16,17}$. No primeiro modelo foram incluídas variáveis distais, representando as dimensões do espaço (município de residência) e do tempo (ano de ocorrência). Em seguida foram acrescidas as variáveis intermediárias, do nível individual, de natureza demográfica (sexo, idade e situação conjugal) e socioeconômica (raça/cor e escolaridade). No terceiro modelo foram introduzidas as variáveis proximais referentes ao contexto em que foi efetuada a certificação das causas dos óbitos, a saber: assistência médica e/ou confirmação diagnóstica na ocasião da morte (definida pela combinação de respostas às variáveis assistência médica, confirmação diagnóstica por exames laboratoriais, cirurgia e necropsia), local de ocorrência, médico atestante e município de ocorrência. Para a variável escolaridade foi criada a categoria "não se aplica”, referente aos óbitos com idades inferiores a seis anos. As demais categorias referem-se ao modelo da DO vigente até 2010, em relação às quais os registros de 2011 em diante foram convertidos pelo SIM ${ }^{18}$. Quanto ao médico atestante, a categoria referente aos serviços de verificação de óbito (SVO) foi desconsiderada devido à inexistência destes serviços no Amazonas.

Os três níveis hierárquicos foram definidos com vistas à caracterização e diferenciação dos fatores distais, explicitados pelas dimensões espacial e temporal e suas variações, relacionadas aos padrões de mortalidade; intermediário, pautado na premissa de vigência de desigualdades sociais em saúde, por meio de variáveis socioeconômicas e demográficas; e proximal, representado por variáveis indicativas das condições relacionadas à assistência médica na ocasião da morte.

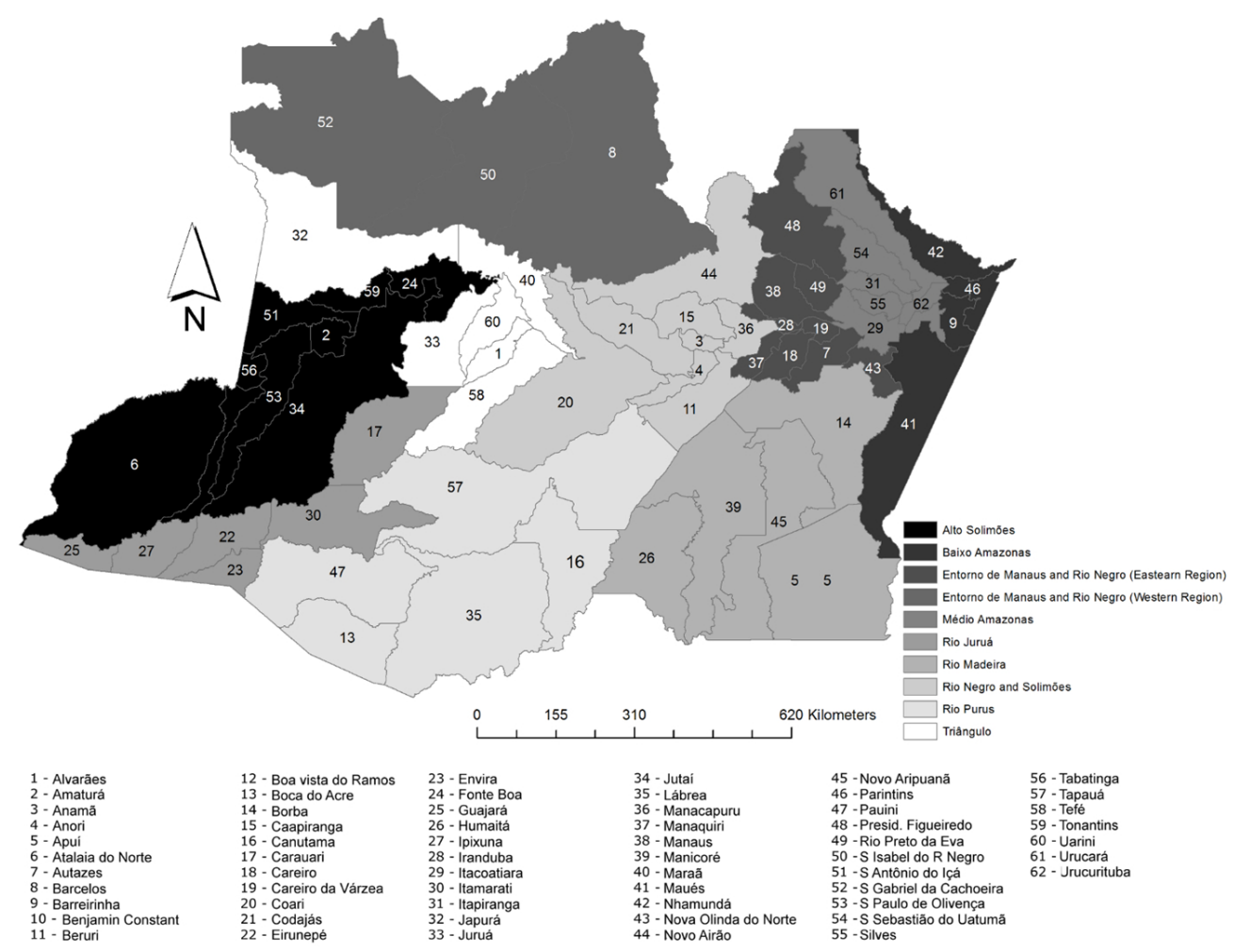

Figura 1. Regiões de saúde e municípios do estado de Amazonas. 
No modelo de regressão logística multinomial as categorias das variáveis explicativas foram inseridas como variáveis indicadoras (dummy) ${ }^{16}$. A inclusão da categoria "ignorado/em branco" teve como intuito verificar a associação entre falhas no preenchimento dos diferentes campos da DO e a qualidade da informação sobre a causa básica do óbito. Para a entrada e retenção das variáveis explicativas no modelo considerou-se a significância estatística da associação com causas inespecíficas e/ou mal definidas nos níveis de $20 \%$ e $5 \%$, respectivamente ${ }^{19}$. A significância estatística foi determinada pelo teste de Wald e a qualidade do ajuste do modelo final pela análise de medidas de deviance ${ }^{19}$. Para todas as análises, foi utilizado o programa Stata $12^{20}$.

O estudo foi aprovado pelo Comitê de Ética em Pesquisa da Fundação Hospital Alfredo da Mata - FUAM.

\section{Resultados}

A população de estudo compreendeu 90.439 registros de óbitos. Entre 2006 e 2012, a mortalidade proporcional por causas mal definidas e inespecíficas no AM foi, respectivamente, 16,6\% e $9,1 \%$. A proporção de mortes por causas mal definidas nas referências de regionais de saúde e nos demais municípios excedeu em cerca de duas e três vezes a da capital, respectivamente. A proporção por causas inespecíficas apresentou pequena variação segundo o tipo de município de ocorrência, correspondendo a 9,1\% no AM. Entre as causas inespecíficas predominaram as causas intermediárias (5,3\%), seguidas pelas não especificadas $(1,9 \%)$ e pelas que não podem ser causa básica $(1,5 \%)$. As causas terminais corresponderam a $0,3 \%$ de todos os óbitos no AM. Embora apresentando proporções distintas em relação ao estado, tal ordenação manteve-se inalterada segundo tipo de município de ocorrência do óbito, exceto nas referências de regionais de saúde, onde as causas que não podem ser causa básica foram mais frequentes que as não especificadas (Tabela 1).

Entre as causas inespecíficas, as categorias "insuficiência cardíaca" (I50), "outras septicemias" (A41) e "hipertensão essencial" (I10) revezaram-se nas três primeiras posições, totalizando de 1 a $2 \%$ de todas as mortes. Proporções mais elevadas de óbitos devido às duas categorias pertencentes às doenças do aparelho circulatório foram observadas fora da capital, onde as septicemias ocuparam a primeira posição. Em relação às causas mal definidas, totalizando quase $15 \%$ no AM sobressaíram-se as "mortes sem assistência" (R98) e as "outras causas mal definidas e as não especificadas de mortalidade" (R99), respectivamente na primeira e segunda posição, exceto na capital onde os postos foram invertidos. Alternaram-se no terceiro posto os "outros sinais e sintomas relativos aos aparelhos circulatório e respiratório" (R09) e a "senilidade" (R54) (Tabela 2).

A chance de causas inespecíficas de morte no AM diminuiu nos dois últimos anos do período analisado. Residir fora da capital mostrou-se positivamente associado às causas inespecíficas, com chances mais elevadas nos municípios que não são referência nas regionais de saúde (Tabela 3).

Tabela 1. Mortalidade proporcional (\%) segundo tipologia da causa básica* e município de ocorrência do óbito, estado do Amazonas, 2006 a 2012.

\begin{tabular}{lcccc}
\hline \multicolumn{1}{c}{$\begin{array}{c}\text { Grupo de } \\
\text { causas }\end{array}$} & \multicolumn{3}{c}{ Município de ocorrência } & Total \\
\cline { 2 - 4 } & Capital & $\begin{array}{c}\text { Referência } \\
\text { regional de saúde }\end{array}$ & $\begin{array}{c}\text { Demais } \\
\text { municípios }\end{array}$ & Amazonas \\
\hline Causas bem definidas & 79,5 & 69,9 & 58,2 & 74,3 \\
Causas mal definidas & 11,6 & 20,6 & 32,6 & 16,6 \\
Causas inespecíficas & 8,9 & 9,5 & 9,2 & 9,1 \\
$\quad$ Causas que não podem ser causa básica & 1,3 & 2,0 & 1,8 & 1,5 \\
Causas intermediárias & 5,4 & 5,5 & 4,6 & 5,3 \\
Causas terminais & 0,2 & 0,4 & 0,7 & 0,3 \\
Causas não especificadas & 2,0 & 1,6 & 2,1 & 1,9 \\
Total & 100,0 & 100,0 & 100,0 & 100,0 \\
\hline
\end{tabular}

${ }^{*}$ Naghavi et al..$^{12}$.

Fonte: Sistema de Informações sobre Mortalidade, Núcleo de Sistemas de Informações em Saúde, Fundação de Vigilância em Saúde do Amazonas - SIM/NUSI/FVS-AM. 
Tabela 2. Mortalidade proporcional (\%) segundo categorias da CID10* mais frequentes, por causas inespecíficas e mal definidas** e tipo de município de ocorrência, estado do Amazonas, 2006 a 2012.

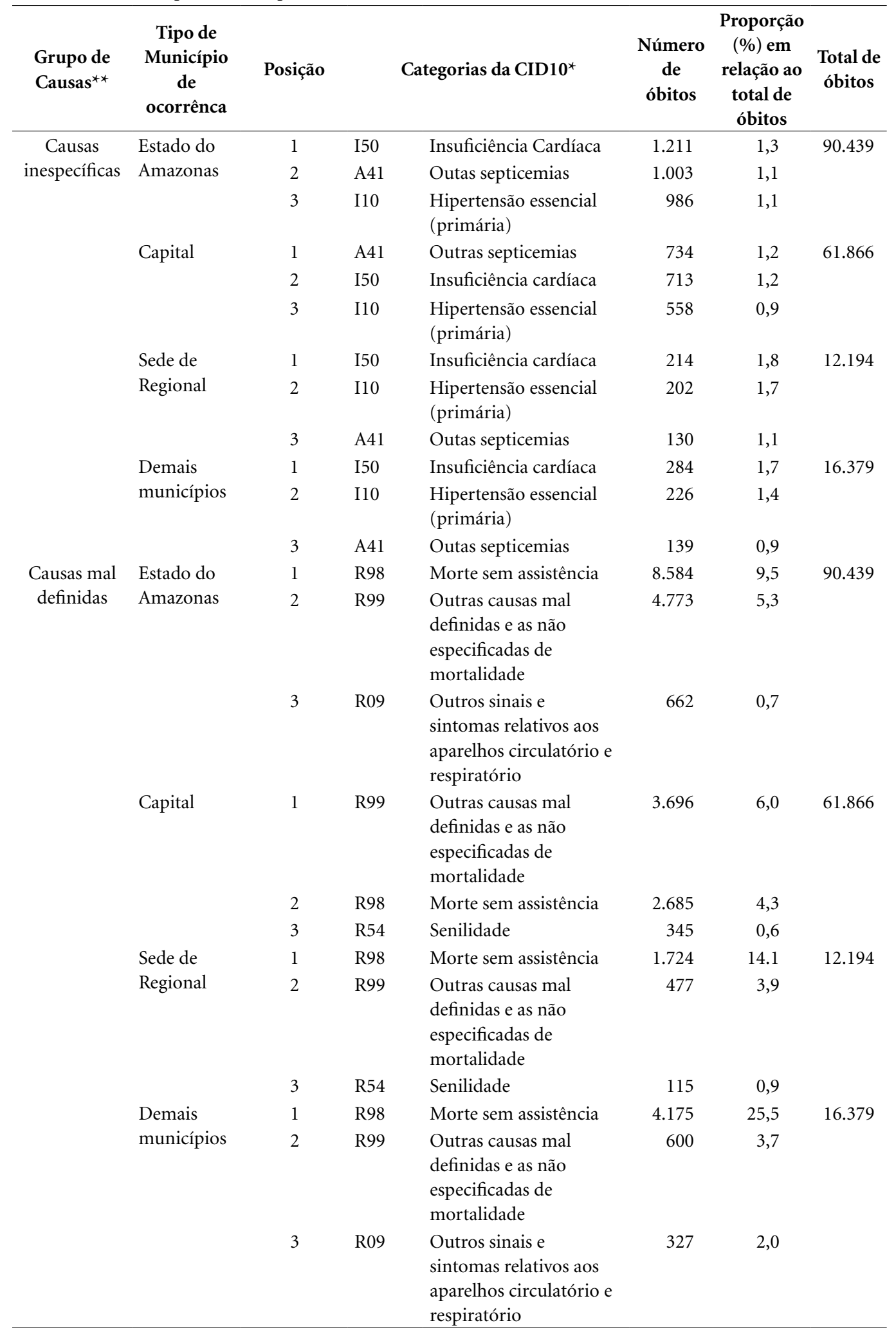

* Classificação Estatística Internacional de Doenças e Problemas Relacionados à Saúde, $10^{\text {a }}$ Revisão. ${ }^{* *}$ Naghavi et al. ${ }^{12}$. Fonte: Sistema de Informações sobre Mortalidade, Núcleo de Sistemas de Informações em Saúde, Fundação de Vigilância em Saúde do Amazonas - SIM/NUSI/FVS-AM. 
Tabela 3. Razões de chances (Odds Ratios-OR) ajustados de causas básicas de óbito inespecíficas e mal definidas ${ }^{*}$ segundo fatores distais, intermediários e proximais, relacionados ao tempo e espaço, de natureza demográfica e socioeconômica e referentes ao contexto em que ocorreu a morte, estado do Amazonas, 2006 a 2012.

\begin{tabular}{|c|c|c|c|c|c|c|}
\hline \multirow{4}{*}{ Nível/Variável } & \multicolumn{6}{|c|}{ Causa básica do óbito } \\
\hline & \multicolumn{3}{|c|}{ Inespecíficas } & \multicolumn{3}{|c|}{ Mal definidas } \\
\hline & \multirow{2}{*}{$\begin{array}{l}\text { OR } \\
0,18\end{array}$} & \multicolumn{2}{|c|}{ IC $95 \%$} & \multirow{2}{*}{$\begin{array}{l}\text { OR } \\
0,03\end{array}$} & \multicolumn{2}{|c|}{ IC 95\% } \\
\hline & & 0,12 & 0,26 & & 0,02 & 0,05 \\
\hline \multicolumn{7}{|l|}{ Distal } \\
\hline \multicolumn{7}{|l|}{ Ano de ocorrência do óbito } \\
\hline 2006 & 1,00 & --- & --- & 1,00 & --- & --- \\
\hline 2007 & 1,00 & 0,91 & 1,09 & 0,86 & 0,80 & 0,91 \\
\hline 2008 & 0,97 & 0,89 & 1,06 & 0,74 & 0,69 & 0,79 \\
\hline 2009 & 0,99 & 0,90 & 1,08 & 0,74 & 0,69 & 0,79 \\
\hline 2010 & 0,96 & 0,88 & 1,04 & 0,68 & 0,63 & 0,72 \\
\hline 2011 & 0,86 & 0,78 & 0,93 & 0,61 & 0,57 & 0,65 \\
\hline 2012 & 0,91 & 0,83 & 0,99 & 0,63 & 0,59 & 0,68 \\
\hline \multicolumn{7}{|l|}{ Município de residência } \\
\hline Capital & 1,00 & --- & --- & 1,00 & --- & --- \\
\hline Sede de regional & 1,20 & 1,13 & 1,28 & 1,67 & 1,59 & 1,76 \\
\hline Demais municípios & 1,37 & 1,29 & 1,45 & 2,84 & 2,73 & 2,96 \\
\hline \multicolumn{7}{|l|}{ Intermediário } \\
\hline \multicolumn{7}{|l|}{ Sexo } \\
\hline Masculino & 1,00 & --- & --- & 1,00 & --- & --- \\
\hline Feminino & 1,10 & 1,05 & 1,16 & 1,02 & 0,98 & 1,06 \\
\hline Em branco/Ignorado & 1,54 & 0,69 & 3,40 & 1,36 & 0,72 & 2,56 \\
\hline \multicolumn{7}{|l|}{ Faixa etária } \\
\hline 0 a 9 anos & 1,00 & --- & --- & 1,00 & --- & --- \\
\hline 10 a 19 anos & 0,55 & 0,43 & 0,72 & 0,82 & 0,64 & 1,06 \\
\hline 20 a 29 anos & 0,34 & 0,26 & 0,44 & 0,65 & 0,50 & 0,83 \\
\hline 30 a 39 anos & 0,44 & 0,34 & 0,56 & 1,04 & 0,82 & 1,33 \\
\hline 40 a 49 anos & 0,71 & 0,55 & 0,90 & 1,49 & 1,17 & 1,89 \\
\hline 50 a 59 anos & 0,84 & 0,66 & 1,07 & 1,93 & 1,52 & 2,44 \\
\hline 60 a 69 anos & 0,92 & 0,73 & 1,17 & 2,03 & 1,61 & 2,58 \\
\hline 70 a 79 anos & 1,01 & 0,80 & 1,28 & 2,61 & 2,06 & 3,30 \\
\hline 80 a 89 anos & 1,22 & 0,97 & 1,55 & 3,76 & 2,98 & 4,76 \\
\hline 90 a 99 anos & 1,37 & 1,06 & 1,75 & 6,07 & 4,77 & 7,71 \\
\hline 100 anos e mais & 1,44 & 0,99 & 2,10 & 7,86 & 5,81 & 10,64 \\
\hline Em branco/Ignorada & 0,73 & 0,41 & 1,30 & 4,87 & 3,14 & 7,55 \\
\hline \multicolumn{7}{|l|}{ Raça/cor da pele } \\
\hline Branca & 1,00 & --- & --- & 1,00 & --- & --- \\
\hline Preta & 1,15 & 0,99 & 1,32 & 1,43 & 1,24 & 1,63 \\
\hline Amarela & 0,77 & 0,52 & 1,14 & 0,96 & 0,65 & 1,42 \\
\hline Parda & 0,91 & 0,85 & 0,96 & 1,97 & 1,86 & 2,08 \\
\hline Indígena & 0,91 & 0,80 & 1,04 & 3,10 & 2,82 & 3,40 \\
\hline Em branco/Ignorada & 0,95 & 0,84 & 1,09 & 1,12 & 0,98 & 1,28 \\
\hline \multicolumn{7}{|l|}{ Situação conjugal } \\
\hline Solteiro & 1,00 & --- & --- & 1,00 & --- & --- \\
\hline Casado & 1,05 & 0,98 & 1,12 & 0,67 & 0,63 & 0,70 \\
\hline Viúvo & 1,00 & 0,93 & 1,09 & 0,66 & 0,62 & 0,70 \\
\hline Separado & 0,94 & 0,78 & 1,12 & 0,61 & 0,51 & 0,72 \\
\hline União estável & 0,86 & 0,71 & 1,03 & 0,82 & 0,72 & 0,93 \\
\hline Em branco/Ignorado & 1,09 & 0,97 & 1,22 & 0,70 & 0,63 & 0,77 \\
\hline
\end{tabular}


Tabela 3. Razões de chances (Odds Ratios-OR) ajustados de causas básicas de óbito inespecíficas e mal definidas $^{\star}$ segundo fatores distais, intermediários e proximais, relacionados ao tempo e espaço, de natureza demográfica e socioeconômica e referentes ao contexto em que ocorreu a morte, estado do Amazonas, 2006 a 2012.

\begin{tabular}{|c|c|c|c|c|c|c|}
\hline \multirow{3}{*}{ Nível/Variável } & \multicolumn{6}{|c|}{ Causa básica do óbito } \\
\hline & \multicolumn{3}{|c|}{ Inespecíficas } & \multicolumn{3}{|c|}{ Mal definidas } \\
\hline & OR & \multicolumn{2}{|c|}{ IC 95\% } & OR & \multicolumn{2}{|c|}{ IC 95\% } \\
\hline \multicolumn{7}{|l|}{ Escolaridade } \\
\hline Sem escolaridade & 1,00 & --- & --- & 1,00 & --- & --- \\
\hline 1a a 4a série & 1,23 & 0,92 & 1,64 & 1,03 & 0,82 & 1,30 \\
\hline 5a a 8a série & 1,09 & 0,82 & 1,45 & 0,93 & 0,74 & 1,17 \\
\hline Médio & 1,03 & 0,78 & 1,38 & 0,75 & 0,59 & 0,94 \\
\hline Superior incompleto & 1,06 & 0,80 & 1,42 & 0,66 & 0,53 & 0,84 \\
\hline Superior completo & 1,09 & 0,80 & 1,47 & 0,49 & 0,38 & 0,64 \\
\hline Não se aplica & 0,48 & 0,34 & 0,70 & 0,88 & 0,64 & 1,22 \\
\hline Em branco/Ignorada & 1,35 & 1,01 & 1,80 & 0,61 & 0,48 & 0,76 \\
\hline \multicolumn{7}{|l|}{ Proximal } \\
\hline \multicolumn{7}{|l|}{ Município de ocorrência } \\
\hline Capital & 1,00 & --- & --- & 1,00 & --- & --- \\
\hline Referência de regional de saúde & 1,16 & 1,01 & 1,34 & 1,50 & 1,20 & 1,86 \\
\hline Demais municípios & 1,31 & 1,16 & 1,48 & 1,52 & 1,29 & 1,79 \\
\hline \multicolumn{7}{|l|}{ Local de ocorrência } \\
\hline Hospital & 1,00 & --- & --- & 1,00 & --- & --- \\
\hline Outros estabelecimentos de saúde & 1,40 & 1,11 & 1,76 & 3,48 & 2,86 & 4,23 \\
\hline Domicílio & 0,86 & 0,80 & 0,92 & 6,29 & 5,86 & 6,74 \\
\hline Via pública & 0,20 & 0,16 & 0,27 & 1,02 & 0,88 & 1,17 \\
\hline Outros & 0,79 & 0,68 & 0,91 & 2,51 & 2,25 & 2,80 \\
\hline Em branco/Ignorado & 1,18 & 0,64 & 2,18 & 4,44 & 2,94 & 6,69 \\
\hline \multicolumn{7}{|l|}{$\begin{array}{l}\text { Assistência médica e/ou confirmação } \\
\text { diagnóstica }\end{array}$} \\
\hline Não & 1,00 & --- & --- & 1,00 & --- & --- \\
\hline Sim & 0,90 & 0,77 & 1,04 & 0,16 & 0,14 & 0,18 \\
\hline Em branco/Ignorado & 1,11 & 0,96 & 1,29 & 0,87 & 0,79 & 0,96 \\
\hline \multicolumn{7}{|l|}{ Médico atestante } \\
\hline Assistente & 1,00 & --- & --- & 1,00 & --- & --- \\
\hline Substituto & 0,99 & 0,93 & 1,05 & 0,99 & 0,88 & 1,11 \\
\hline IML & 0,48 & 0,43 & 0,53 & 7,16 & 6,49 & 7,90 \\
\hline Outros & 0,82 & 0,76 & 0,88 & 1,51 & 1,38 & 1,66 \\
\hline Em branco/Ignorado & 0,83 & 0,75 & 0,92 & 9,18 & 8,42 & 10,02 \\
\hline
\end{tabular}

* Naghavi et al. ${ }^{12}$. ${ }^{* *}$ OR: Odds Ratio; IC95\%: Intervalo de 95\% de confiança.

Fonte: Sistema de Informações sobre Mortalidade, Núcleo de Sistemas de Informações em Saúde, Fundação de Vigilância em Saúde do Amazonas - SIM/NUSI/FVS-AM.

Entre os óbitos das mulheres a chance de causas inespecíficas foi $10 \%$ mais elevada em comparação aos dos homens. Não foi observada associação com ausência de informação sobre o sexo. As idades entre 10 e 49 anos mostraram-se associadas a chances menores de causas inespecíficas, em especial dos 20 aos 29 anos, em que a redução foi de aproximadamente $65 \%$ em re- lação à faixa de 0 aos 9 anos. A cor parda foi a única entre as categorias de raça/cor da pele a apresentar associação, com redução de cerca de $10 \%$ em relação à cor branca. Quanto à escolaridade, as categorias "não se aplica" e "ignorado/ em branco" mostraram-se associadas a chances, respectivamente, $52 \%$ menores e $37 \%$ mais elevadas em relação ao grupo que nunca frequentou 
escolas. Não se observou associação com situação conjugal (Tabela 3 ).

A ocorrência da morte fora da capital aumentou a chance de mortalidade por causas inespecíficas em cerca de $16 \%$ e $31 \%$, respectivamente nas referências de regionais de saúde e nos demais municípios. Em relação aos óbitos hospitalares a chance de causas inespecíficas foi 1,4 vezes maior quando as mortes ocorreram em outros estabelecimentos de saúde, e menor entre os óbitos domiciliares (14\%), ocorridos em outras localizações (21\%) e, principalmente, em vias públicas (80\%). Comparativamente aos médicos assistentes, as chances de causas inespecíficas foram cerca de $50 \%$, 18\% e $17 \%$ menores quando os óbitos foram atestados, respectivamente, por legistas do Instituto Médico Legal (IML), por outros médicos e quando não havia informação sobre o médico atestante. O preenchimento da DO por médicos substitutos assim como o registro de assistência médica não interferiram na chance de mortalidade por causas inespecíficas (Tabela 3).

A chance de mortalidade por causas mal definidas diminuiu ao longo do período analisado. Em relação a 2006, a redução foi em torno de $15 \%$ em 2007, 25\% em 2008 e 2009, e entre 30\% e $40 \%$ a partir de 2010 . Residir nas referências de regionais de saúde e nos demais municípios elevou em 1,7 e 2,8 vezes a chance de causas mal definidas, respectivamente, em relação à capital (Tabela 3).

Não se observou associação com o sexo. A respeito da idade, em relação à primeira década de vida, a chance de óbito por causas mal definidas diminuiu entre os 20 e 29 anos em 35\%. Chances mais elevadas e de maneira crescente foram observadas a partir dos 40 anos chegando a alcançar valores aproximadamente quatro, seis e oito vezes mais altos nas faixas de 80 a 89,90 a 99 e 100 anos e mais, respectivamente, em relação à de 0 a 9 anos. A ausência do registro da idade resultou em chance quase cinco vezes maior de causa mal definida. Quando assinaladas na DO, as categorias de raça/cor da pele preta, parda e indígena mostraram-se associadas a chances cerca de $40 \%$, $100 \%$ e $210 \%$ vezes maiores, respectivamente, quando comparadas à cor branca. Em relação à condição de solteiro, reduções da ordem de 30 a $40 \%$ foram observadas para as categorias "casado", "viúvo", "separado" e na falta de informação sobre a situação conjugal, e de 18\% para "união estável". A chance de causas mal definidas variou inversamente em relação ao nível de escolaridade, a partir da categoria "ensino médio" até "superior completo". A escolaridade não informada resultou em chance $40 \%$ mais baixa (Tabela 3).

Morrer fora da capital, independentemente do tipo de município, implicou em chances 1,5 vezes mais altas de causas mal definidas. Falecer fora dos hospitais, exceto quando em via pública, também mostrou-se associado a chances mais elevadas, 6,29 vezes maior entre os óbitos domiciliares. A falta da informação sobre o local de ocorrência do óbito aumentou cerca de 4,4 vezes a chance de causas mal definidas. $O$ registro de assistência médica e/ou confirmação diagnóstica na ocasião da morte reduziu em $84 \%$ a chance de causas mal definidas, ao passo que o preenchimento da declaração de óbito por legistas do IML e por outros médicos a elevou em aproximadamente 7,2 e 1,5 vezes, respectivamente. Nas declarações sem informação sobre o médico atestante, a chance foi 9,2 vezes maior em relação às certificadas pelo médico assistente (Tabela 3 ).

\section{Discussão}

Em média, cerca de um em cada quatro óbitos de residentes no AM e ocorridos no próprio estado entre 2006 e 2012 apresentou algum grau de indeterminação no registro da causa básica no SIM, com predomínio das causas mal definidas em relação às inespecíficas. Dos fatores investigados, apenas situação conjugal e assistência médica não apresentaram associação com a ocorrência de causas inespecíficas, e sexo com a de mal definidas.

As causas mal definidas se concentraram em duas categorias, mortes sem assistência, notadamente fora da capital, e as outras causas mal definidas e as não especificadas de mortalidade, a mais frequentemente certificada em Manaus. O predomínio das duas categorias, assim como as respectivas ordenações inversas em Manaus e fora da capital, também foram observados em análise da mortalidade na população idosa brasileira $^{21}$. Em Belo Horizonte, MG, as outras causas mal definidas e as não especificadas de mortalidade (R99) foram certificadas em 5\% das mortes entre 2011 e $2013^{22}$. Quanto às causas inespecíficas mais frequentes no AM entre 2006 e 2012, a hipertensão essencial (I10) e as septicemias não especificadas (A41.9) apresentaram proporções semelhantes em Belo Horizonte ${ }^{22}$ e a insuficiência cardíaca (I50) se destacou entre as mortes de idosos brasileiros ${ }^{21}$.

Os resultados por ora descritos devem ser interpretados à luz das relações entre os fatores associados à indeterminação da causa de morte, 
concebidas segundo o modelo de regressão hierárquica formulado. No nível distal, as dimensões do tempo e do espaço refletem a influência das políticas e ações de saúde implementadas sobre o sistema de saúde e as informações sobre mortalidade.

A qualidade das informações sobre causas de morte na capital foi melhor do que no restante do estado. Variação semelhante foi reportada por Kanso et al. ${ }^{21}$ para a população idosa no AM. Ainda assim, a mortalidade proporcional por causas mal definidas observada em Manaus é elevada, tanto no presente estudo como por outros autores, situando-se em torno de $12 \%$, quatro vezes acima da média nacional e a mais alta entre as capitais brasileiras $^{22}$.

As causas inespecíficas ocorreram em menor frequência comparativamente às mal definidas no AM, e com pouca variação entre a capital e os demais municípios, resultados estes similares aos observados para a população idosa do estado em $2007^{21}$. Estimativa mais elevada $(18,5 \%)$ foi reportada por Ishitani et al. ${ }^{22}$ entre 2011 e 2013, mais que o dobro do verificado no presente estudo, possivelmente devido a diferenças metodológicas na seleção dos códigos da CID10.

A redução da ocorrência de causas mal definidas foi acompanhada pela queda do sub-registro de óbitos no Amazonas, de 21,0\%, em 2006, para $13,4 \%$, em $2012^{23}$. Embora somente em 2011 e 2012 a ocorrência de causas inespecíficas também declinou. Resultados de diferentes estudos baseados em metodologias distintas convergem no sentido do aumento da cobertura do SIM e da melhoria da qualidade da informação sobre causas de morte nas últimas décadas em todo o país, com avanços expressivos nos estados das regiões Norte e Nordeste em períodos mais recentes ${ }^{24,25}$. Investigações adicionais são necessárias para esclarecer em que medida a melhoria da informação ocorreu em decorrência da captação de óbitos inicialmente não registrados - redução do sub-registro - acompanhada pela determinação das causas básicas e/ou se a investigação dos óbitos registrados sem causa definida resultou na recuperação da informação sobre a causa básica.

O aprimoramento do SIM é resultado das iniciativas empreendidas nas últimas décadas com vistas à qualificação dos registros contínuos de mortalidade no Brasil ${ }^{25,26}$. Todavia, a elevada mortalidade proporcional por causas mal definidas no AM observada no presente estudo, assim como por outros autores no mesmo período, reforça a necessidade de investimentos contínuos com vistas à sua redução, assim como do sub-re- gistro e da irregularidade das informações. Em 10\% dos municípios do AM a mortalidade proporcional por causas mal definidas ficou acima de 47,2\% entre 2008 e 2010, configurando a pior situação entre as unidades da federação ${ }^{25}$.

A maior cobertura da rede de saúde, assim como a concentração da assistência de maior complexidade em Manaus podem servir como explicação para a elevação das chances de indeterminação do registro da causa básica do óbito fora da capital. O aumento das chances de causas inespecíficas e mal definidas entre as referências de regionais de saúde e os demais municípios, e destes em relação à capital, possivelmente reflete as variações estruturais da rede de atenção em saúde. Em especial, a associação entre causas mal definidas e residência fora da capital, mais intensa nos municípios que não são referências regionais, pode ser indicativa da existência de restrições de oferta de e/ou acesso a serviços de saúde $^{27}$, localizados em regra - quando existentes - nas sedes municipais. No AM, acresce-se ainda as longas distâncias a serem percorridas, usualmente por via fluvial e sujeitas, frequentemente, a limitações de ordem sazonal e financeira ${ }^{28}$.

Reforçam a suposição acerca da relação com a restrição de oferta de e/ou acesso a serviços de saúde as elevadas parcelas de "mortes sem assistência" (R98) e por "outras causas mal definidas e as não especificadas de mortalidade" (R99) ${ }^{29}$, assim como as expressivas elevações da chance de causas mal definidas nas DO não preenchidas por médicos - nas situações em que não há médico na localidade e no momento da ocorrência da morte - e entre os óbitos ocorridos fora de hospitais, exceto em vias públicas, assim como a sua intensa redução frente a existência de assistência médica e/ou confirmação diagnóstica na ocasião da morte. Já as causas inespecíficas, por serem caracterizadas pelos diagnósticos incompletos, pressupõem a certificação do óbito por profissionais médicos, o que pode explicar a ausência de associação com a existência, ou não, de assistência médica no momento do óbito. Relações semelhantes entre ocorrência hospitalar e qualidade da certificação de causas de morte foram observadas em Belo Horizonte, $\mathrm{MG}^{22}$, assim como para a população idosa brasileira ${ }^{21}$.

Ajustadas pelas variáveis distais, as associações identificadas no nível intermediário expressam as relações entre desigualdades demográficas e socioeconômicas e a qualidade das informações sobre mortalidade por causas.

A relação entre mortalidade por causas definidas e idade está de acordo com o relatado a 
partir de diferentes estudos realizados no país. Entre as possíveis explicações salienta-se a dificuldade de estabelecer uma causa básica frente a concomitância de múltiplas morbidades, situação comum entre os idosos ${ }^{30,31}$.

A redução das chances de causas inespecíficas nas faixas de idade entre 10 e 49 anos e no sexo masculino podem estar associadas à ocorrência de mortes por causas não naturais, sabidamente mais elevada entre homens adultos jovens ${ }^{32}$. As causas externas tendem a ser mais bem definidas em relação às naturais, seja no momento da certificação ou no da codificação, quando se costuma dispor de informações adicionais a respeito das circunstâncias do acidente ou violência que produziu a lesão fatal, registradas em boletins de ocorrência policial ou acessíveis em jornais e outros meio de divulgação, como a internet ${ }^{33,34}$.

A associação entre mortalidade por causas mal definidas e fatores socioeconômicos, como cor da pele não branca e baixa escolaridade, também foi relatada por diferentes autores ${ }^{21,31,35}$, refletindo situações de exclusão e restrição de acesso a serviços de saúde. Diferentemente do observado no AM, em tais estudos verificou-se também excesso de mortalidade sem determinação da causa em mulheres, mas não em relação à situação conjugal.

Em comparação à condição de solteiro, todas as categorias de situação conjugal associaram-se a chances menores de causas mal definidas. No caso da união estável, a magnitude da redução foi intermediária quando comparada às categorias casado, viúvo e separado. Deve ser considerada a influência, a partir de 2010, quando a opção união estável foi introduzida na $\mathrm{DO}$, do possível deslocamento de registros anteriormente assinalados como solteiro ${ }^{18}$.

A associação entre a condição de solteiro e risco mais elevado de causas mal definidas também foi reportada em estudo de coorte ocupacional realizado nos EUA, atribuída ao isolamento frente à inexistência de laços parentais, e intensificada com a ocorrência do óbito em domicílio, sem a presença de outras pessoas ${ }^{36}$. Todavia, a associação entre mortalidade e a situação de solteiro parece não se restringir às causas mal definidas, conforme relatos de outros estudos ${ }^{37,38}$. Padrões comportamentais específicos assim como o impacto potencial das consequências psicológicas relacionadas à condição de solteiro foram aventados como possíveis explicações ${ }^{38}$.

Na população idosa brasileira as causas inespecíficas, ainda que em menor grau, mostraramse associadas à raça/cor da pele não branca e baixa escolaridade ${ }^{21}$, em desacordo com os resultados para o AM. Entre as possíveis explicações para os resultados discordantes observados, há que se considerar as diferenças metodológicas entre os dois estudos, como as populações alvo e os períodos analisados, assim como as distintas definições de causas inespecíficas utilizadas. Por outro lado, estruturas de confundimento poderiam justificar a inexistência de associação com indicadores de nível socioeconômico como, por exemplo, a maior concentração de população indígena nos municípios que não são referências regionais.

Após ajustadas segundo os níveis distal e intermediário, as associações com as variáveis proximais indicam a influência da assistência médica e/ou confirmação diagnóstica sobre a qualidade dos registros de mortalidade.

O aumento da chance de causas mal definidas frente à certificação do óbito pelo IML está possivelmente relacionado à inexistência de serviços de verificação de óbito (SVO) no AM. No estado de São Paulo a menor mortalidade proporcional por causas mal definidas nos municípios com SVO foi explicada pela elevada frequência de realização de necropsias entre os óbitos avaliados inicialmente como devidos a causas naturais, resultando no esclarecimento da causa mortis em mais de $90 \%$ das vezes ${ }^{39}$. Em João Pessoa, PB, a implantação do SVO foi seguida por redução de $78 \%$ dos óbitos sem causa definida ${ }^{40}$. Em Pernambuco a contribuição dos SVO, além do esclarecimento das causas de mortes, estendeu-se para o aprimoramento da vigilância epidemiológica por meio da notificação oportuna de doenças de notificação compulsórias, mortes maternas, fetais e infantis ${ }^{41}$. Todavia, a relação custo-efetividade da implantação de uma rede de SVO no Amazonas deve ser considerada, especialmente nos municípios que não a capital, caracterizados por baixa densidade demográfica, longas distâncias das sedes municipais e dificuldades de locomoção.

A ampliação da rede de atenção primária por meio da estratégia de saúde da família (ESF), em curso em todo o país, pode contribuir para a melhoria dos registros de causas de morte ${ }^{26,42}$. Uma vez realizados os princípios da integralidade e da continuidade do cuidado à saúde pelas equipes de saúde da família, especialmente se amparadas por uma rede de serviços de apoio diagnóstico e terapêutico ambulatoriais e hospitalares, esperar-se-ia o aprimoramento da capacidade de diagnóstico pelo sistema de saúde. Somada à implantação de uma rede de SVO, a expansão da ESF traz consigo o potencial para uma melhor 
certificação das causas de morte, em especial frente a ocorrência de óbitos domiciliares e nos municípios que não são referências regionais no $\mathrm{AM}$, em grande parte desprovidos de coberturas adequadas de assistência médica.

As coberturas insuficientes da ESF verificadas no AM durante o período por ora analisado cerca de 32\% em Manaus e $48 \%$ nos municípios restantes ${ }^{43}$, podem explicar, ao menos em parte, a elevação das chances de causas mal definidas entre as mortes ocorridas em domicílio e fora da capital. Por sua vez, a implantação da investigação de óbitos por causas mal definidas ${ }^{44}$ assim como a descentralização da coordenação do SIM para os Distritos Sanitários Especiais Indígenas (DSEI) ${ }^{45}$, podem ter contribuído para a redução das causas mal definidas observada a partir de 2006. Todavia, investigações adicionais são necessárias para avaliar a participação efetiva dessas ações para a qualificação do SIM no AM e em todo o país.

Considerando que o presente estudo utilizou dados do sistema de informação sobre mortalidade é importante ressaltar que elementos como sub-registro e incompletude podem interferir nos resultados, tendo em vista as suas prováveis naturezas não aleatórias ${ }^{46}$. A associação positiva entre incompletude dos campos referentes a diferentes variáveis explicativas selecionadas no presente estudo e causas mal definidas sugere que a má certificação das causas do atestado médico de óbito tende a ser acompanhada por um preenchimento inadequado das DO de maneira mais ampla. Resultados semelhantes foram observados na população idosa brasileira conforme relatado por Kanso et al. ${ }^{21}$. O comprometimento insuficiente dos médicos no preenchimento da DO, em especial das causas de morte, remete à necessidade de sensibilização e capacitação permanentes, com início à época da graduação $0^{8,43}$. Há que se considerar, entretanto, a influência de aspectos de natureza gerencial sobre a ocorrência de erros, assim como de incompletudes, tais como as mudanças no modelo da DO e as atualizações do SIM, e de infraestrutura, relacionados à transmissão em rede dos dados.

O presente estudo apresenta limitações, entre as quais a natureza secundária dos registros de óbitos utilizados. Em especial, a validade da informação sobre a causa básica da morte pode ser comprometida de diferentes formas, seja por falhas no processo de diagnóstico, de certificação na declaração de óbito, de codificação ${ }^{47}$, de processamento e de transferência de bases de dados. No caso do AM, o sub-registro de óbitos, embora em declínio, era ainda expressivo no período analisado no presente estudo. Os resultados, portanto, devem ser entendidos como restritos ao subconjunto dos óbitos registrados no SIM.

\section{Conclusão}

Os achados do presente estudo apontam para uma tendência de melhoria da qualidade da informação sobre causa de morte no AM de 2006 a 2012, apesar da elevada mortalidade proporcional por causas sem determinação da causa básica ainda verificada. A distribuição das mortes por causas mal definidas e inespecíficas não ocorreu aleatoriamente, mostrando-se associada às dimensões espaço-temporais, a fatores demográficos e socioeconômicos e à assistência médica na ocasião do óbito.

Em que diz respeito aos fatores associados à mortalidade por causas mal definidas e inespecíficas, o modelo de predição aqui proposto deve ser considerado pelo sistema de saúde em seus diferentes níveis com vistas ao aprimoramento da qualidade dos registros de causas de morte no AM. Ressalta-se a importância do desenvolvimento de modelos de análise específicos para os diferentes cenários socioeconômicos, demográficos e de saúde, considerando a escassez de estudos a respeito da qualidade das informações sobre mortalidade, voltados para a realidade da região Norte do Brasil.

A relevância dos registros de mortalidade de boa qualidade deve ser permanentemente enfatizada nos cursos de graduação em medicina e por meio de iniciativas voltadas à educação continuada, destacando-se a responsabilidade do médico pelo correto preenchimento da declaração de óbito. Conceitos relativos ao registro de causas de morte, tais como causa básica, intermediária, terminal, contribuinte e associada devem ser periodicamente revistos e discutidos, sempre que possível, à luz das experiências cotidianas acumuladas ao longo da prática médica. 


\section{Colaboradores}

PCS Balieiro, LCF Silva, VS Sampaio, EX Monte e EMS Pereira participaram da concepção do estudo, análise e interpretação dos dados e redação do artigo. LAF Queiroz e R Saraiva colaboraram na análise e interpretação dos dados e redação do artigo. AJL Costa participou da concepção do estudo, análise e interpretação dos dados, redação e revisão do artigo.

\section{Agradecimentos}

A Ana Alzira Cabrinha, gerente do NUSI/FVS AM, pela cessão do banco de dados e apoio ao desenvolvimento deste trabalho.

À Fundação de Amparo à Pesquisa no Estado do Amazonas (FAPEAM) pelo financiamento do Projeto "Fortalecimento das atividades de pesquisa, tecnologia e/ou inovação para execução de Programas Estaduais de Prevenção e Controle de Doenças" (Programa Estratégico de Ciência, Tecnologia \& Inovação nas Fundações Estaduais de Saúde).

\section{Referências}

1. Laurenti R, Mello-Jorge MHP. Atestado de óbito: aspectos médicos, estatísticos, éticos e jurídicos. São Paulo: Cremesp; 2015.

2. Danilova I, Shkolnikov VM, Jdanov DA, Meslé F, Vallin J. Identifying potential differences in cause- ofdeath coding practices across Russian regions. Popul Health Metr 2016; 14:8.

3. Gilbert NL, Fell DB, Joseph KS, Liu S, León JA, Sauve R. Temporal trends in sudden infant death syndrome in Canada from 1991 to 2005: Contribution of changes in cause of death assignment practices and in maternal and infant characteristics. Paediatr Perinat Epidemiol 2012; 26(2):124-130.

4. Garces RG, Sobel HL, Pabellon JAL, Lopez Jr JM, Castro MQ, Nyunt-U S. A comparison of vital registration and reproductive-age mortality survey in Bukidnon, Philippines, 2008. Int J Gynecol Obstet 2012; 119(2):121-124.

5. França E, Abreu DX, Rao C, Lopez AD. Evaluation of cause-of-death statistics for Brazil, 2002-2004. Int J Epidemiol 2008; 37(4):891-901.

6. Lima EEC, Queiroz BL. A evolução do sub-registro de mortes e causas de óbitos mal definidas em Minas Gerais: diferenciais regionais. Rev Bras Est Pop 2011; 28(2):303-320.

7. World Health Organization (WHO). International Classification of Diseases (ICD) [Internet]. 2016. [acessado 2016 Nov 10]. Disponível em: http://apps.who. int/classifications/icd10/browse/2016/en

8. Jorge MHPM, Cascão AM, Reis AC, Laurenti R. Em busca de melhores informações sobre a causa básica do óbito por meio de linkage: um recorte sobre as causas externas em idosos - Estado do Rio de Janeiro, Brasil, 2006. Epidemiol Serv Saude 2012; 21(3):407418.

9. Foreman KJ, Lozano R, Lopez AD, Murray CJ. Modeling causes of death: an integrated approach using CODEm. Popul Health Metr 2012; 10:1.

10. Lima CRA, Schramm JMA, Coeli CM, Silva MEM. Revisão das dimensões de qualidade dos dados e métodos aplicados na avaliação dos sistemas de informação em saúde. Cad Saude Publica 2009; 25(10):2095-2109.

11. Instituto Brasileiro de Geografia e Estatística (IBGE). Atlas do Censo Demográfico 2010. Rio de Janeiro: IBGE. 2013.

12. Naghavi M, Makela S, Foreman K, O’Brien J, Pourmalek F, Lozano R. Algorithms for enhancing public health utility of national causes-of-death data. Popul Health Metr 2010; 8:9.

13. GBD 2013 Mortality and Causes of Death Collaborators. Global, regional, and national age-sex specific all-cause and cause-specific mortality for 240 causes of death, 1990-2013: a systematic analysis for the Global Burden of Disease Study 2013. Lancet 2014; 385(9963):117-171.

14. Amazonas. Secretaria de Estado de Saúde. Resolução CIB que dispõe sobre a revisão do desenho regional do estado do Amazonas para a saúde [Internet]. 2011. [acessado 2016 Nov 10] Disponível em: http://www. saude.am.gov.br/cib/docs/res_cib_2011_059.pdf 
15. Motta E, Kerr LRFS. Medidas de ocorrência de doenças, agravos e óbitos. In: Almeida Filho N, Barreto ML, organizadores. Epidemiologia \& Saúde. Fundamentos, Métodos, Aplicações. Rio de Janeiro: Guanabara Koogan; 2011. p. 95-117.

16. Kleinbaum DG, Kupper LL, Nizam A, Rosenberg ES Applied regression analysis and other multivariable methods. Toronto: Nelson Education; 2013.

17. Victora CG, Huttly SR, Fuchs SC, Olinto MT. The role of conceptual frameworks in epidemiological analysis: a hierarchical approach. Int J Epidemiol 1997; 26(1):224-227.

18. Brasil. Ministério da Saúde (MS). Saúde Brasil 2012: uma análise da situação de saúde e dos 40 anos do Programa Nacional de Imunizações. Brasília: Editora do Ministério da Saúde; 2014.

19. Hosmer DW. Applied logistic regression. $3^{\text {rd }}$ ed. New Jersey: John Wiley \& Sons; 2013.

20. StataCorp. Stata Statistical Software: Release 12. College Station: StataCorp; 2011.

21. Kanso S, Montilla DER, Leite IC, Moraes EN. Diferenciais geográficos, socioeconômicos e demográficos da qualidade da informação da causa básica de morte dos idosos no Brasil. Cad Saude Publica 2011; 27(7):1323-1339.

22. Ishitani LH, Teixeira RA, Abreu DMX, Paixão LMMM, França EB. Qualidade da informação das estatísticas de mortalidade: códigos garbage declarados como causas de morte em Belo Horizonte, 2011-2013. Rev Bras Epidemiol 2017; 20(Supl. 1):34-45.

23. Departamento de Informática do SUS (Datasus). Indicadores e dados básicos do Brasil [Internet]. 2013. [acessado 2016 Nov 22]. Disponível em: http://tabnet. datasus.gov.br/cgi/idb2012/matriz.htm

24. Queiroz BL, Freire FHMA, Gonzaga MR, Lima EEC. Estimativas do grau de cobertura e da mortalidade adulta $(45 \mathrm{q} 15)$ para as unidades da federação no Brasil entre 1980 e 2010. Rev Bras Epidemiol 2017; 20(Supl. 1):21-33

25. Frias PG, Szwarcwald CL, Lira PIC. Avaliação dos sistemas de informações sobre nascidos vivos e óbitos no Brasil na década de 2000. Cad Saude Publica 2014; 30(10):2068-2280.

26. Lima EEC, Queiroz BL. Evolution of the deaths registry system in Brazil: associations with changes in the mortality profile, under-registration of death counts, and ill-defined causes of death. Cad Saude Publica 2014; 30(8):1721-1730.

27. Campos D, França E, Loschi RH, Souza MFM. Uso da autópsia verbal na investigação de óbitos com causa mal definida em Minas Gerais, Brasil. Cad Saude Publica 2010; 26(6):1221-1233.

28. Amazonas. Secretaria de Estado de Saúde. Plano diretor de regionalização do estado do Amazonas [Internet]. 2003 [acessado 2016 Nov 22]. Disponível em: http://www.saude.am.gov.br/index.php?id=pdr

29. Cunha CC, Teixeira R, França E. Avaliação da investigação de óbitos por causas mal definidas no Brasil em 2010. Epidemiol. Serv. Saude 2017; 26(1):19-30.
30. Martins Junior DF, Costa TM, Lordelo MS, Felzemburg RDM. Tendência dos óbitos por causas mal definidas na região Nordeste do Brasil, 1979-2009. Rev Assoc Med Bras 2011; 57(3):338-346.

31. Cascão AM, Jorge MHPM, Costa AJL, Kale PL. Uso do diagnóstico principal das internações do Sistema Único de Saúde para qualificar a informação sobre causa básica de mortes naturais em idosos. Rev Bras Epidemiol 2016; 19(4):713-726.

32. Campos MEAL, Ferreira LOC, Barros MDA, Silva HL Mortes por homicídio em município da Região Nordeste do Brasil, 2004-2006 a partir de dados policiais. Epidemiol Serv Saude 2011; 20(2):151-159.

33. Villela LCM, Rezende EM, Drumond EF, Ishitani LH, Carvalho GML. Utilização da imprensa escrita na qualificação das causas externas de morte. Rev Saude Publica 2012; 46(4):730-736.

34. Soares Filho AM, Cortez-Escalante JJ, França E. Revisão dos métodos de correção de óbitos e dimensões de qualidade da causa básica por acidentes e violências no Brasil. Cien Saude Colet 2016; 21(12):3803-3818.

35. Fiorio NM, Flor LS, Padilha M, Castro DS, Molina MCB. Mortalidade por raça/cor: evidências de desigualdades sociais em Vitória (ES), Brasil. Rev Bras Epidemiol 2011; 14(3):522-530.

36. Cragle DL, Fletcher A. Risk factors associated with the classification of unspecified and/or unexplained causes of death in an occupational cohort. Am J Public Health 1992; 82(3):455-457.

37. Fouillet A, Rey G, Laurent F, Pavillon G, Bellec S, Ghihenneuc-Jouyaux C, Clavel J, Jougla E, Hémon D. Excess mortality related to the August 2003 heat wave in France. Anne Int Arch Occup Environ Health 2006; 80(1):16-24.

38. Bos V, Kunst AE, Keij-Deerenberg IM, Garssen J, Mackenbach JP. Ethnic inequalities in age- and cause-specific mortality in The Netherlands. Int J Epidemiol 2004; 33(5):1112-1119.

39. Rozman MA, Eluf-Neto J. Necropsia e mortalidade por causa mal definida no Estado de São Paulo, Brasil. Rev Panam Salud Publica 2006; 20(5):307-313.

40. Sales Filho R. Análise da implantação do Serviço de Verificação de Óbitos de João Pessoa - PB no Sistema de Informação sobre Mortalidade [tese]. Recife: Centro de Pesquisas Aggeu Magalhães; 2011.

41. Azevedo BAS, Vanderlei LCM, Mello RJV, Frias PG Avaliação da implantação dos Serviços de Verificação de Óbito em Pernambuco, 2012: estudo de casos múltiplos. Epidemiol Serv Saude 2016; 25(3):595-606.

42. Szwarcwald CL, Frias PG, Souza Junior PRB, Almeida WS, Morais Neto OL. Correction of vital statistics based on a proactive search of deaths and live births: evidence from a study of the North and Northeast regions of Brazil. Popul Health Metr 2014; 12:16.

43. Brasil. Ministério da Saúde (MS). Departamento de Atenção Básica. Portal do Departamento de Atenção Básica. [Internet]. 2016. [acessado 2016 Set 26]. Disponível em: http://dab.saude.gov.br/portaldab/ 
44. França E, Teixeira R, Ishitani L, Duncan BB, Cortez -Escalante JJ, Morais Neto OL, Szwarcwald CL. Causas mal definidas de óbito no Brasil: método de redistribuição baseado na investigação do óbito. Rev Saude Publica 2014; 48(4):671-681.

45. Brasil. Ministério da Saúde (MS). Portaria no 116, de 11 de fevereiro de 2009. Regulamenta a coleta de dados, fluxo e periodicidade de envio das informações sobre óbitos e nascidos vivos para os Sistemas de Informações em Saúde sob gestão da Secretaria de Vigilância em Saúde. Diário Oficial da União 2009; 12 fev.

46. França EB, Cunha CC, Vasconcelos ANM, Escalante JJC, Abreu DX, Lima RB, Morais Neto OL. Avaliação da implantação do programa "Redução do percentual de óbitos por causas mal definidas" em um estado do Nordeste do Brasil. Rev Bras Epidemiol 2014; 17(1):119-134.

47. Hernández B, Ramírez-Villalobos D, Romero $\mathrm{M}$, Gómez S, Atkinson C, Lozano R. Assessing quality of medical death certification: Concordance between gold standard diagnosis and underlying cause of death in selected Mexican hospitals. Popul Health Metr 2011; 9:38.

Artigo apresentado em 05/04/2017

Aprovado em 02/03/2018

Versão final apresentada em 04/03/2018 